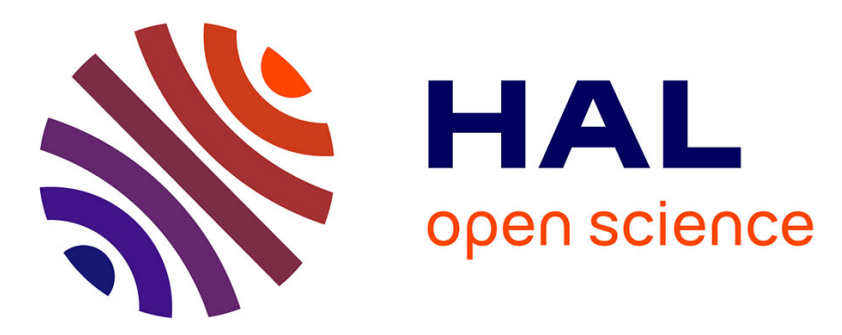

\title{
Underwater Object Tracking Using Time Frequency Signatures of Acoustic Signals
}

Angela Digulescu, Ion Candel, Cornel Ioana, Diana Bucur, Teodor Petrut

\section{To cite this version:}

Angela Digulescu, Ion Candel, Cornel Ioana, Diana Bucur, Teodor Petrut. Underwater Object Tracking Using Time Frequency Signatures of Acoustic Signals. OCEANS 2014 - OCEANS '14 MTS/IEEE. Ocean Regeneration, Apr 2014, Taipei, Taiwan. pp.978-1-4799-3646-5/14. hal-00985765

\section{HAL Id: hal-00985765 https://hal.science/hal-00985765}

Submitted on 30 Apr 2014

HAL is a multi-disciplinary open access archive for the deposit and dissemination of scientific research documents, whether they are published or not. The documents may come from teaching and research institutions in France or abroad, or from public or private research centers.
L'archive ouverte pluridisciplinaire HAL, est destinée au dépôt et à la diffusion de documents scientifiques de niveau recherche, publiés ou non, émanant des établissements d'enseignement et de recherche français ou étrangers, des laboratoires publics ou privés. 


\title{
Underwater Object Tracking Using Time Frequency Signatures of Acoustic Signals
}

\author{
Digulescu Angela ${ }^{1}$, Candel Ion ${ }^{2}$, Ioana Cornel $^{2}$, Bucur Diana ${ }^{3}$, Petrut Teodor ${ }^{2}$ \\ ${ }^{1}$ Faculty of Military Electronic and Information Systems, Military Technical Academy, Bucharest, Romania \\ adigulescu@mta.ro \\ ${ }^{2}$ Gipsa-lab, Grenoble Institute of Technology, Grenoble, France \\ \{ion.candel, cornel.ioana, teodor-ion.petrut\}@gipsa-lab.grenoble.inp.fr \\ ${ }^{3}$ Power Engineering Faculty, University POLITEHNICA of Bucharest, Romania \\ diana.bucur@upb.ro
}

\begin{abstract}
Detecting underwater objects is an important application in marine applications. Most of the techniques are based on the amplitude related techniques, whereby the amplitude of the received echo is used to detect objects within specified bounds. Amplitude techniques however are prone to interference and attenuation, thus limiting the capabilities of such systems. Hence, the aim of this paper is to propose a new technique that detect and track underwater moving objects using the turbulence generated by the object. Wideband signals have proven to be a very efficient alternative for merging turbulent flow characteristics and waveform design in order to describe and explain the behavior of turbulence, both artificial and natural. Therefore, constructing adapted waveforms to the natural turbulence embedded in the flow, as well as to the artificial turbulence created by an unknown underwater moving object may hold the key for a new technique for underwater object tracking. When acoustic signals with a particular Instantaneous Frequency Law traveling into underwater environment will hit a moving object, their Instantaneous Frequency Law will capture the object's artificial turbulence, as well as the natural turbulence embedded in the flow. Experimental results carried out in our reduced scale facility provide the validation of the technique.
\end{abstract}

Keywords-wide band signals; turbulence; instantaneous frequency law; object detection; underwater acoustics

\section{INTRODUCTION}

Tracking underwater objects in an active context represents an ongoing challenge from the signal processing point of view. The echoes reflected by the target are analyzed by the receiver, in order to detect the target's presence. This analysis is based on the estimation of the time of flight of the reflected signal in water, therefore an amplitude one. Amplitude techniques however have a number of drawbacks that limit the tracking performance. First, amplitude techniques are prone to interference from several factors: ambient noise, electronic noise, other sources interference and subsequent echoes from previous emissions. Since the technique is based on the time of flight estimation, using cross-correlation, all of these factors negatively influence the overall correlation strength and coefficient. A weak correlation will provide biased results in terms of target's position. Wideband signals are then used because they are more robust. Furthermore, since underwater objects exhibit a stronger signature in the time - frequency plane, it is therefore preferable to exploit the time frequency signature of the received signal, rather than the amplitude one. In order to achieve such a thing, the emitted waveform must be adapted to the underwater context, namely the turbulence left by the tracked object. This is done by an appropriate wave forming technique that will boost the interaction between the turbulent signature of the underwater object and the emitted acoustic wave.

The paper is structured as follows: section 2 presents the model corresponding to the interaction between the acoustic waves and the turbulence associated to the underwater object. Here, we show that is the signal's envelope can be modified using a polynomial phase in order to increase the interferences with turbulence produced by the underwater object. Section 3 will present the associated signal processing algorithms that allow the precise extraction of the phase in order to quantify the changes on the received phase. Section 4 will present the experimental validation of the method in our reduced scale experimental facility, and section f5 will present the conclusions of further developments of our work.

\section{ADAPTIVE WAVEFORMS BASED ON INSTANTANEOUS FREQUENCY LAWS}

A simple model of signal propagation in an underwater environment is based on the transformations suffered by the received signal. This signal is a attenuated and delayed version of the emitted signal. Let $s(t)$ be the emitted signal and $r(t)$ the received signal. The relation between these two signals is:

$$
\begin{aligned}
& s(t)=A_{\text {em }}(t) \cdot \exp \left[j \cdot \omega_{0} \cdot t\right] \\
& r(t)=\alpha(t) \cdot s(t-\tau),
\end{aligned}
$$

where $\omega_{0}=2 \cdot \pi \cdot f_{0}$ corresponds to the resonance frequency of the transducers, $\alpha(t)$ is the attenuation coefficient of the signal in water, and $\tau$ is the time of flight between the emitter and the receiver. It is becoming clear very early that the effects of underwater signal interference are not separable in the 
amplitude domain and therefore a spectral-based approach is needed.

Thanks to the work of Kolmogorov described in [1] and [2], the turbulence manifests itself in the low region of the spectrum. This region is usually found between 0 and $10 \mathrm{kHz}$ and does not generally interact with the region corresponding to the acoustic transducer frequencies used in underwater tracking. It is clear that the interference between the two regions (turbulent and acoustic is very weak). Moreover, the turbulence phenomena is a wide band one rather than a narrow band one, as the acoustic signals used by current techniques, as illustrated in Fig. 1.

The choice for the acoustic frequency of the transducers represents a tradeoff: low frequencies mean a low attenuation coefficient, but limit the range of application by introducing long echoes; high frequencies mean that echoes are short, but the high attenuation coefficient means a reduced detector range. It is then obvious that an amplitude technique is not able to bring the two fields (turbulent and acoustic) together and therefore the only way is to use the frequency information.

Tests have shown that the received signal frequency does not change with turbulence, because of the gap between the turbulence spectrum and the acoustic signal spectrum. However, the shape of the envelope does change, hence its frequency. It is therefore necessary to act on the shape of the envelope, rather than on the carrier.

Conventional techniques described in [3] use standard short duration narrowband pulses and are modulated by the underwater environment. Since the signal detector at the receiving side is amplitude based one, the information embedded in the envelope spectrum is discarded, but it may hold the key to a more robust tracking of the underwater target (object). Amplitude modifications on the envelope represent the turbulent signature associated with the wakes generated by the underwater target. Since the emitted pulse has a short duration, this signature is not fully developed on the envelope and thus little can be said about the target's characteristics.

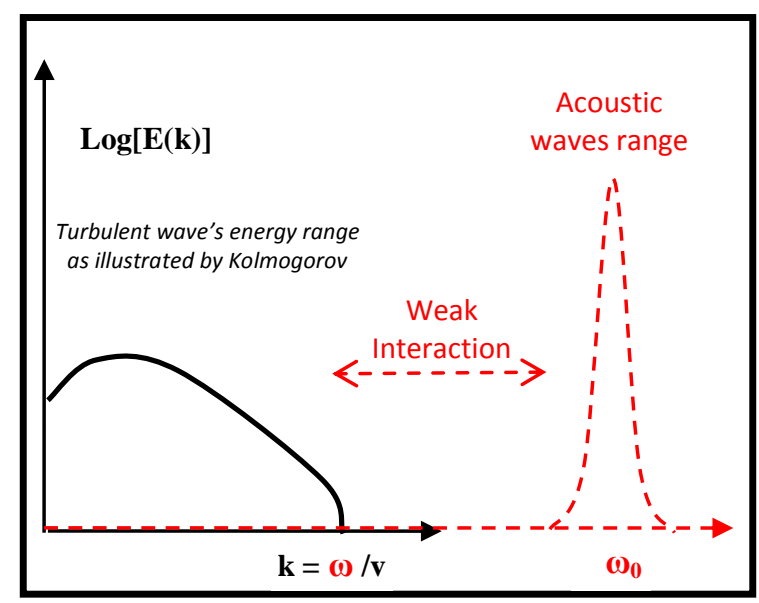

Fig. 1. Low interaction between acoustic and turbulent waves.

Moreover, even with a long duration narrowband pulse, the signatures embedded in the envelope are not sufficiently developed and prone to interference. The reason behind this drawback is that envelope - turbulence interaction is manifested on an amplitude scale, not on a frequency one.

Therefore, by increasing the duration of the emitted signal and changing the shape of the envelope, the waveforms used for underwater tracking are far more adapted to the context. The next question is what shape the envelope should take in order to boost the signature trace of the turbulence.

Since the turbulence occupies a spectrum region that can be estimated using Kolmogorov theory based equations [4], it is then possible to modulate the envelope content into a frequency modulation in the turbulence's bandwidth. By doing this, the detector will search the frequency modifications induced by the turbulence on the envelope's Instantaneous Frequency Law (IFL).

There are numerous IFL shapes that the envelope can have, flat, linear, cubic, etc. Among all these shapes, only one is best suited to boost the interaction with turbulence and in order to discover the appropriate shape, the envelope phase at the emission and reception side must be taken into account. The emitted and received envelopes can be written as:

$$
\begin{aligned}
& A_{e}(t)=\exp \left[j \cdot \phi_{e}(t)\right] \\
& A_{r}(t)=\exp \left[j \cdot \phi_{r}(t)\right]
\end{aligned}
$$

where $\phi_{e}(t)$ and $\phi_{r}(t)$ are the phases of $A_{e}(t)$ and $A_{r}(t)$, the emitted and received envelopes. In order to overlap the turbulence spectrum on the envelope spectrum, the emitted phase can be best described using an analytical polynomial form:

$$
\phi_{e}(t)=a_{0}+a_{l} \cdot t+\sum_{k=2}^{N} a_{k} \cdot t^{k},
$$

where the terms $a_{0}, a_{1}$ and $a_{k}$ are the coefficients of the polynomial phase. The $a_{1}$ coefficient translates the acoustic signal bandwidth from the high acoustic range into the low turbulence range. However, since the turbulent spectrum is a wide band one and the translated acoustic bandwidth is a narrowband one, additional terms must be introduced in the polynomial formula of the phase in order for the translated wave to cover the entire range of the turbulence spectrum. This is done by the summation terms $a_{k} t^{k}$ in (3). The choice for the polynomial degree $N$ in (3) is done by modeling the received phase:

$$
\phi_{r}(t)=a_{0}+a_{1} \cdot t+\sum_{k=2}^{N} a_{k} \cdot t^{k} \cdot\left(1-\frac{u_{\text {target }}}{c_{\text {si gnal }}}\right)^{k},
$$

where $u_{\text {target }}$ is the target velocity underwater and $c_{\text {signal }}$ is the acoustic signal velocity in water, approximately $1500 \mathrm{~m} / \mathrm{s}$. The received phase formula in (4) shows that the modifications on the IFL induced by the target's wakes indroduce a term in the received phase dependent on the target velocity and signal velocity. However, this term is very small because the target velocity is low compared to the signal velocity. This is why with the conventional techniques relying on narrowband short duration pulses) these changes were not visible. In order to boost the effect of this term, the polynomial degree must be higher than one. 


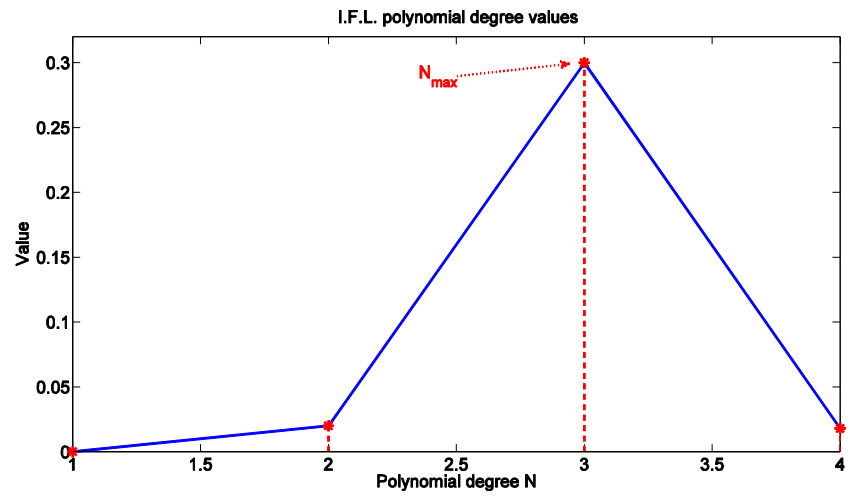

Fig. 2. The choice for the optimum polynomial degree.
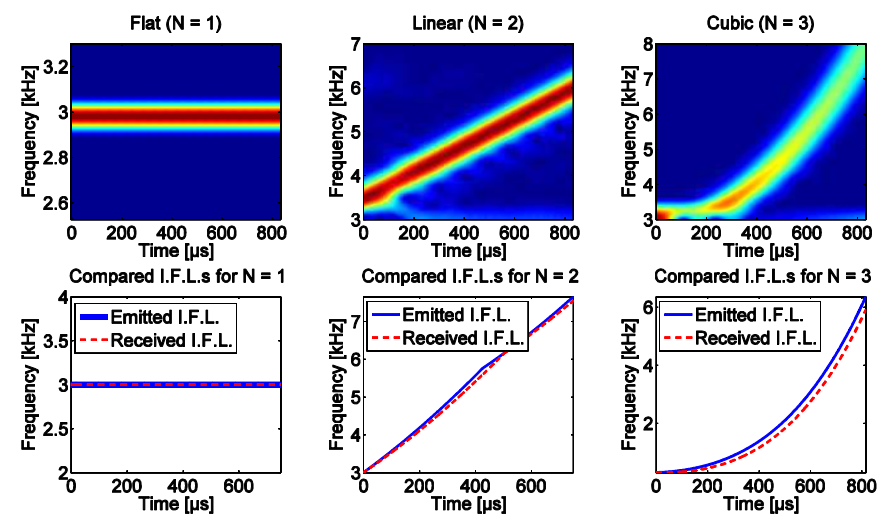

Fig. 3. The choice for the optimum polynomial degree as depicted by experimental methods.

Simulation tests have shown that the value of $N$ that maximizes the impact of the $u_{\text {target }} / c_{\text {signal }}$ term is three, as illustrated in figure 2. The impact value for $N$ roses up to the value 3 and it starts decreasing. It is therefore optimum to adopt a cubic modulation of the phase, rather than a constant or linear one. The choice for $N=3$ was confirmed also in experimental conditions detailed in section 4 , as illustrated in figure 3 .

Figure 3 shows three cases of IFL transmissions: constant (no modulation), linear and cubic modulations. Each emitted law was compared with its corresponding received law. The comparisons showed that the most significant changes are observed for cubic IFLs and therefore the most suited modulation of the phase is a cubic one. Having both theoretical and experimental validations, the shape of the emitted waveform will be described by:

$$
s(t)=\underbrace{\exp \left(j \cdot \omega_{0} \cdot t\right)}_{\text {carrier }} \cdot \underbrace{\exp \left(\sum_{k=0}^{3} a_{k} \cdot t^{k}\right)}_{\text {envelope }}
$$

The shape of the envelope in (5), as well as its corresponding frequency law is illustrated in fig. 4. Fig. 4 shows the IFL of the emitted signal. A similar IFL is computed at the receiving side, and therefore a robust signal
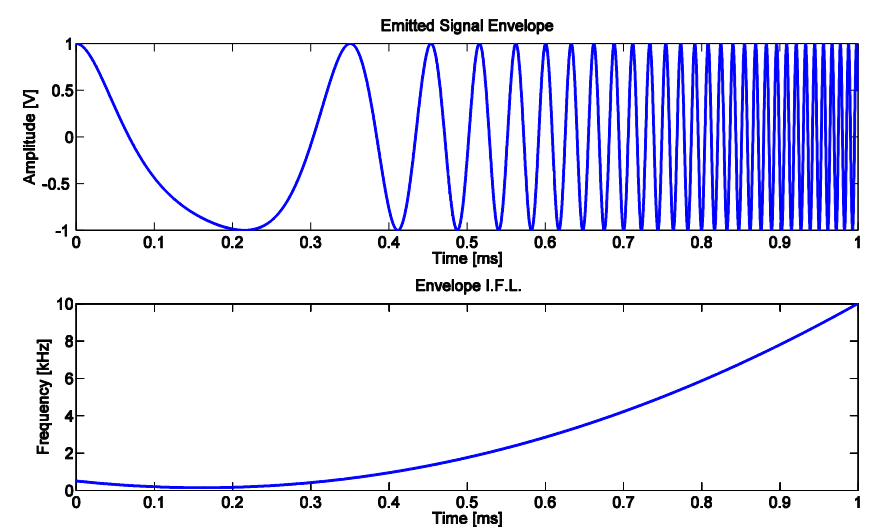

Fig. 4. The emitted signal envelope and its corresponding IFL

processing algorithm has to be developed in order to recover the IFL as accurately as possible, without interference.

\section{INSTANTANEOUS FREQUENCY LAW ESTIMATION ALGORITHM}

Previous sections have established that the standard signal waveform required for a more robust underwater tracking needs to be replaced with a wideband signal representing a carrier with an envelope characterized by a particular IFL.

The estimation of IFL represents the most sensitive part of the tracking method. The most common method used for IFL visualization and estimation is based on the signal spectrogram. The method computes the argument of column maxima of the spectrogram performed on the envelope signal, as described in (6):

$$
\operatorname{IFL}(t)=\arg \max [\operatorname{STFT}(t, f)],
$$

where $\operatorname{STFT}(t, f)$ is the time frequency representation of the envelop obtained using the spectrogram.

This technique, although simple and easy to implement has a major drawback: if the signal's time-frequency presents artifacts with a higher energy than the local IFL elements, the trace corresponding to the estimated IFL will be deviated to the local contaminated maxima, as illustrated in figure 5. Here, a possible drawback in the estimation of IFLs is shown: local relevant IFL content is affected by components in the same bandwidth as the envelope, leading to a wrong IFL estimation. Being outside the local range of the IFL, these components are difficult to filter.

Recently, a solution can be developed by working around these components. In [6] and [7], the authors present a new approach that uses the coherence of local phase of the components. The method is based on the modeling of frequency content in overlapping sliding windows on the input signal. According to [7], for each signal window, $w$, the local time-frequency content is estimated using local linear frequency modulated signals within the duration and bandwidth of the signal piece in the analyzed window. These linear frequency modulations represent approximations that best fit the time frequency content of the signal in window $w$. 


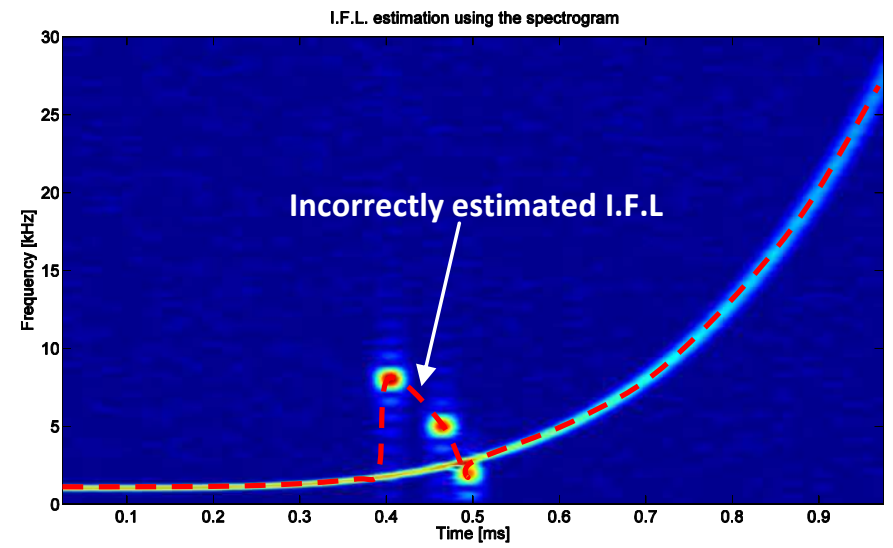

Fig. 5. Unreliable IFL estimation using the spectrogram.

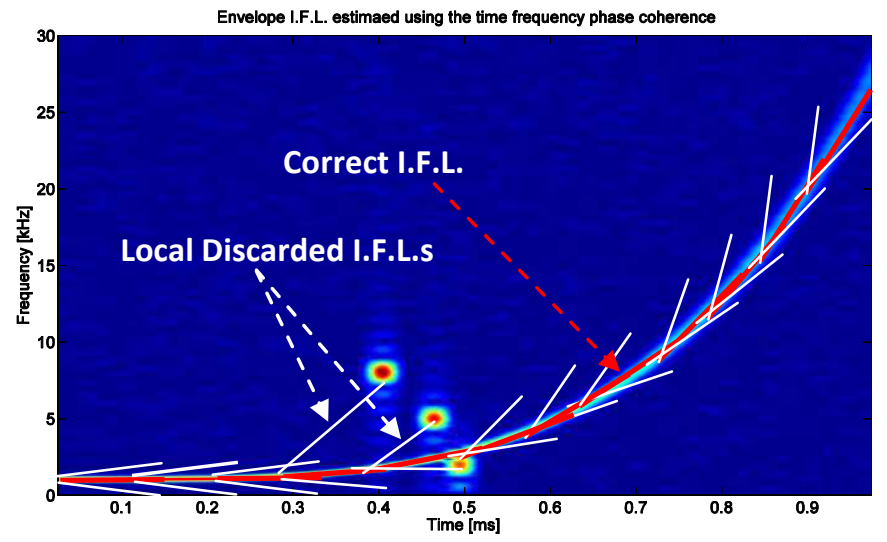

Fig. 6. Robust IFL estimation using the time - frequency - phase coherence of local linear frequency modulated components.

This is done by using the ambiguity function (7) in order to generate the linear frequency modulations that could be part of the global IFL. The ambiguity function is calculated for each window $w$ of the analyzed signal:

$$
f_{A m b}^{(w)}(t, f)=\sum_{n=-\infty}^{\infty} s_{w}(n) \cdot s_{w}^{*}(n+\tau) \cdot \exp (-j \cdot 2 \cdot \pi \cdot n \cdot f),
$$

where $s_{w}$ is the part of the analyzed signal found in the window $w$ and $\tau$ is the time lag needed to compute the ambiguity function. The result of the ambiguity function represents a parameter that defines a chirp signal which approximates the signal present in the window, $s_{w}$.

The choice between which local IFL has to be chosen is done by computing the coherence with the IFLs in adjacent windows. In this way, only the local IFLs that are coherent with the cubic shape of the IFL are kept and the other IFLs corresponding to frequency contamination are discarded, as illustrated in fig. 6 . The resulting IFL will be a reunion of local IFLs as depicted by:

$$
\operatorname{IFL}(t)=\bigcup_{k=1}^{N} I F L_{l o c}^{w}(t),
$$

where $I F L_{l o c}{ }^{w}$ is the IFL computed for the signal in window $w$. the coherence between the local IFLs is based on the hypothesis that if two adjacent IFLs are in the same time -
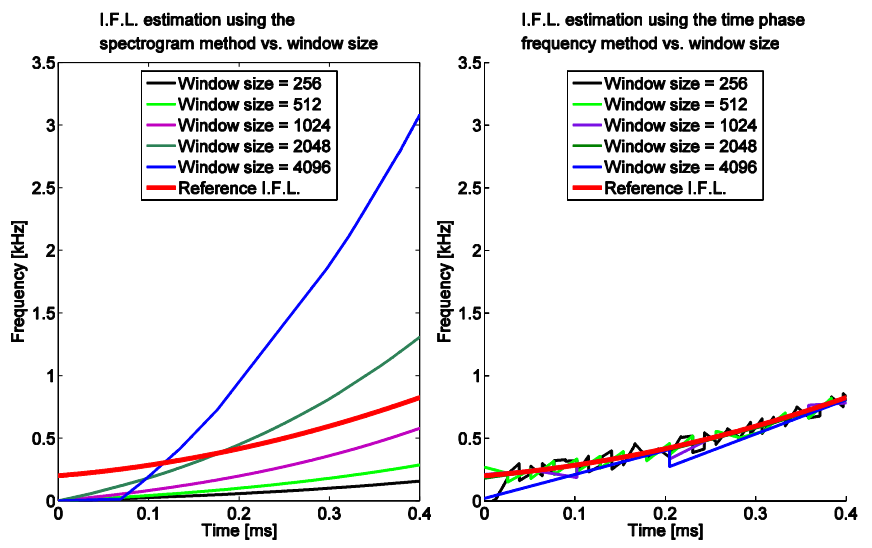

Fig. 7. IFL estimation using the spectrogram and time - frequency - phase coherence methods vs. window size.

frequency region, then the two IFLs can be merged and thus belonging to the global IFL [7].

However, since both methods (spectrogram and time frequency - phase) methods perform signal windowing, the unwanted effects associated with this operation can bias the results. It is well known that the spectrogram method suffers from the Heisenberg uncertainty principle [5]. A test of robustness to the signal windowing reveals the influence of the window length on the overall IFL curve, as illustrated in fig. 7. The results show that the I.FL.s estimated with the spectrogram for several sizes of windows are very far apart from the reference IFL. On the other hand, I.F.Ls issued from the time - frequency - phase method are closer to the reference. This is due to the local coherence of IFLs that minimizes the effects of the signal windowing. Once the received IFL is computed, it is compared with the emitted IFL (from the emitted envelope). Their difference will highlight whether the object has passed or not.

\section{RESULTS}

The experimental validation of the underwater object tracking was carried out in a reduced scale experimental facility. A reduced-scale submarine was placed in flow and wideband signals containing second order IFL were transmitted. The received echo's IFL was estimated using the time - frequency - phase algorithm. Since most of the unwanted noise and interference is found outside the useful bandwidth, the extracted IFL is affected mostly by the turbulent pattern of the moving object.

Several received "pings" from the object should provide a trajectory, changes in velocity, as well as information about the type and size of the vessel. Fig. 8 shows the difference between the emitted and received IFLs. It is interesting to see also the succession of received IFLs from static (no object in range) and dynamic (object in range) conditions. The best rendering of the two situations is achieved by plotting the difference between the emitted and received IFLs. This is useful in the case of objects generating weak signatures, such as the case in our reduced scale facility. The results can be seen in fig. 9. Note that in order to prove the concept the number of emissions for the dynamic configurations is higher than for the static one. 


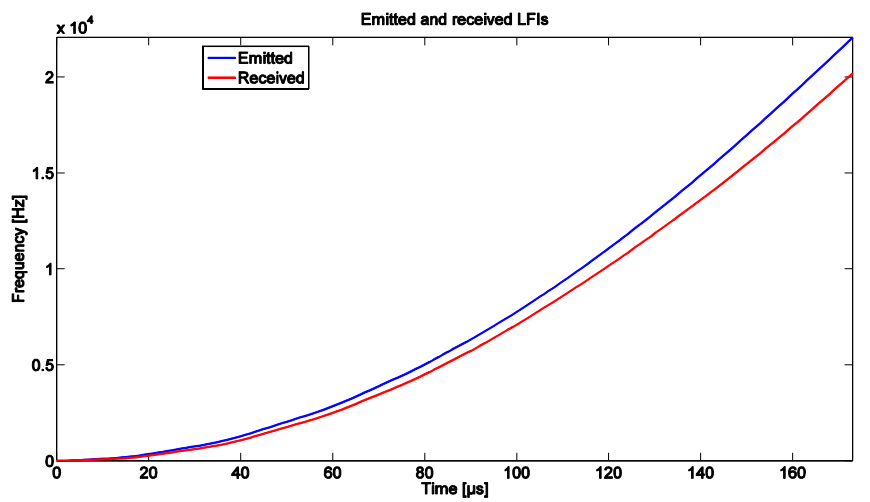

Fig. 8. The distinction between received and emitted IFLs for an underwater object tracking.

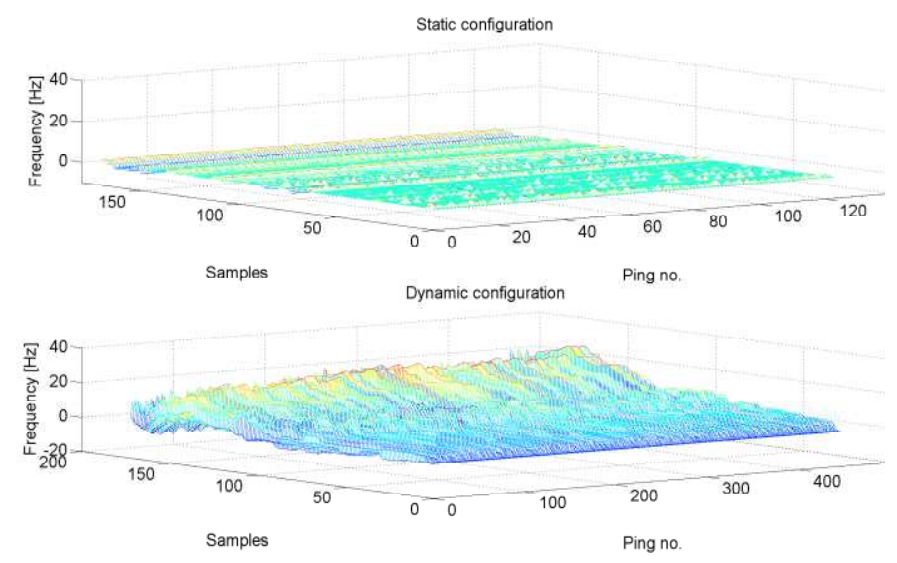

Fig. 9. Rendering of IFL modifications for static vs. dynamic configurations.

Since we are dealing with real signals, the IFL modifications for the static configurations are not zero because of the uneven distribution of noise in the spectrum which changes for each ping. Due to the non-steady characteristics of the noise, the signatures are not consistent to a deterministic one, such as the case for the dynamic situation. Here, the signature changes for each ping converge to the hypothesis of an artificial moving object. A distributed system, composed by several acoustic paths defined as illustrated in the figure 10, will allow the tracking of the object in the area of interest.

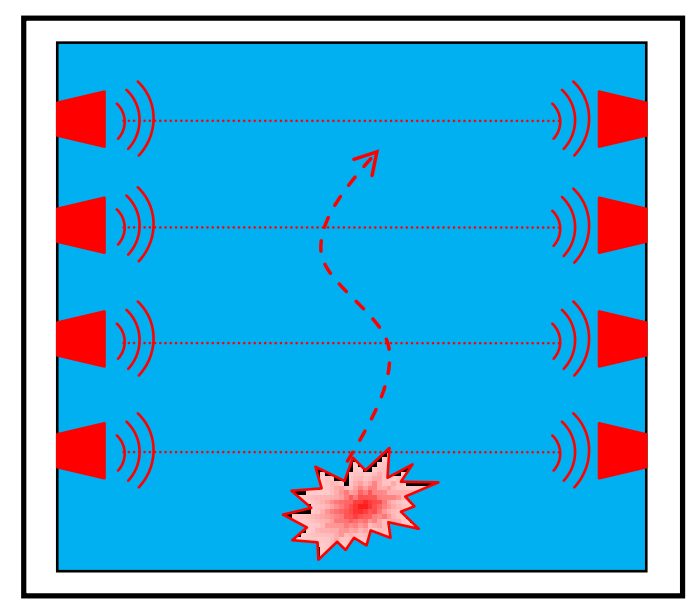

Fig. 10. Distributed signal processing on a multipath configuration.

\section{CONCLUSIONS}

This technique is part of the adapted waveforms approach for acoustic signals developed in a particular underwater situation and goes beyond conventional systems for underwater turbulence estimation. Combining previous concepts such as the Kolmogorov theory for turbulent flows and advanced signal processing techniques such as time phase - frequency methods, this physically - driven technique is more appropriate for highly turbulent environments.

One of the most important contributions of this paper is the concept of adapting acoustic signals to underwater turbulent environments. This relies on replacing the narrowband short acoustic pulses used by current techniques with wide band signals containing particular IFLs that are best suited for underwater object tracking.

Future works will concentrate on experimental validation in a real context using larger targets on a wide applicative range. Also, the definition of a distributed processing system is an extension that will allow us the tracking of the object in a given area.

\section{ACKNOWLEDGMENT}

This work has been supported, in part, by the French DGA-DGCIS RAPIDE project "GREENAR".

\section{REFERENCES}

[1] U. Frisch, Turbulence, the Legacy of A. N. Kolmogorov, Cambridge : Cambridge University Press, 1995.

[2] J. Jimenez, "The Contributions of A.N. Kolmogorov to the Theory of Turbulence”, Arbor, Vol. 704, pp. 589-606

[3] R. Jiwani, S. Lucas, Nivus France. "Methods for Flow Measurement". Sessenheim, 2002.

[4] G. Ahmadi, Turbulence. Potsdam, NY : Clarkson University, 2006.

[5] I. Candel, et al, "Construction et Analyse des Signaux NonStationnaires Adaptés à la Caractérisation des Phénomènes Turbulents",.Brest, France, 2013. XXIVème Colloque Gretsi.

[6] F. Dadouchi, et al. "Distributed network of underwater acoustic sensors based on local time-frequency cherence analysis", San Diego, Proceedings of OCEANS 2013.

[7] C. Ioana et al., "Time-Frequency-Pahse Tracking Approach: Application to Underwater Signals in a Passive Context", IEEE International Conference on Acoustics, Speech and Signal Processing, Dallasa, U.S.A., 2010. 